\title{
A New Generation: How refugee trauma affects parenting and child development
}

\author{
Arq boekenreeks 2013, ISBN 9789089532671, by Elisa van Ee
}

\section{Johanna Hermansson Tham, MD*}

Elisa van Ee's dissertation $A$ New Generation: How refugee trauma affects parenting and child development is well written, important and interesting to read. The dissertation focuses on the relationship between the traumatization of refugee parents and the impact traumatization has on their non-traumatized children.

Van Ee is a clinical psychologist and psychotherapist affiliated with Centrum 45, the Dutch national institute for specialist diagnostics and treatment of psycho-trauma resulting from persecution, war and violence.

The author begins with a thorough review of literature on how parents' traumatization affect their non-traumatized children. She continues to give us a critical review of the research studies done concerning the designs, measuring instruments, samples used and their limitations.

Van Ee's clear-sighted reasoning in her dissertation is the result of a combination of deep clinical knowledge and recent research results including her own dissertation. The combined approach and perspective on trauma and attachment theory is a new and

\footnotetext{
*) Red Cross Centre for Tortured Refugees, Stockholm, Sweden
}

Correspondence to: johanna.hermansson@tele2.se rewarding way of looking at very complex processes and how to understand how PTSD-symptoms can be transmitted to children who themselves have not been subjected to trauma.

This is an important issue to address since there is a gap in knowledge and in understanding for these processes according to Van Ee. For example, the understanding of why certain dyads are affected and others are not, and also the understanding of the mechanisms involved in the transmission of traumatization and how to treat severe traumatized parents and their children.

Besides our limited knowledge in intergenerational transmission processes, there is a lack of research covering this area. Most studies done have been retrospective in nature, and there is also a lack of studies done concerning the parent-child perspective.

Focusing on the interaction parent-child, is a useful way of increasing our understanding of the processes mentioned. This is exactly what Elisa van Ee has contributed to and formulated in an excellent manner in her dissertation.

The parents in her study have experienced a whole array of trauma including torture, time in prison, physical injury, exposure to war/combat situations, and rape and have witnessed relatives and friends being murdered. 
The number of participants, asylum seekers or refugee parents, in different studies, ranged from 49 in samples with only mothers, to 80 in studies with both mothers and fathers. The children were between the ages of 18-42 months. They were born in the Netherlands and had not been exposed to any trauma. The mothers ranged in age from 19-44 years.

In chapter 3 maternal traumatization as a risk factor for child development is examined. The interrelations between maternal posttraumatic stress symptoms, mother-child interaction and infants' psychosocial functioning is analyzed.

A mother's emotional availability influences how the relation between motherchild develops. A relation is a reciprocal process and the less emotionally available the mother is, the less responsive the child. The interaction of emotional availability mother/ child was assessed with Emotional Availability Scale (EAS) an instrument developed by Biringen. EAS measures the following dimensions: sensitivity, structuring, nonintrusiveness and non-hostility in the adult and responsiveness and involvement in the child. The attachment pattern of the child was also assessed.

From Van Ee's studies we learn that there is a connection between mothers' PTSD symptoms and childrens' psychosocial functioning. Mothers with more symptoms of posttraumatic stress showed less emotional accessibility in the relationship with their child. The less sensitivity the mother expressed, the less response the child showed.

In this work we have contact with research never done before. For the first time the quality of the interaction between traumatized refugee fathers and their children was observed. No gender differences were found between severe trauma- tized mothers and severe traumatized fathers in the quality of parent-child interaction. Gender did not predict EAS scores on any of the four scales. The study found traumatized refugee mothers and refugee fathers to be equally sensitive, structured, intrusive and hostile. Although there were differences in the quantity of interaction between mothers and fathers and their children. (Chapter 4).

Another study that illustrates a topic not discussed much before is the situation of children born of wartime rape. Van Ee discuss the urgent perspective on the issue of trauma and parenting by describing the harrowing situation of children born as a result of wartime rape.

To illustrate the complexity of the matter I'd like to quote an interview with a little girl. The girl was born as a result of rape and when describing the relationship with her mother she concluded: "mother is all about pain and suffering, and my hugs bring her even more pain.... I know she loves me and hates me at the same time."

From this research, we know that traumatized refugee parents as well as many traumatized children have the capability of functioning well despite the adversity they have lived through. Elisa van Ee brings up the question of resilience in refugee families as a way of understanding this. Resilience regarded as an individual's own capacity to adapt, to cope and withstand hard circumstances. There are, of course, other issues that are important for refugee families living in exile.

Van Ee proposes that both refugee mothers and refugee fathers ought to be included in the treatment. She recommends a combined treatment for mothers, fathers and children as a successful way to work with the interaction of parent and child. Elisa van Ee presents the method used at Centrum 45, multifamily group therapy, MFT" it works 
well for refugee families coping with traumatic stress and parenting difficulties " and she emphasizes the group setting as very successful because the group gives a feeling of hope, and the families can share their experiences. (Chapter 5).

This is an impressive work by Elisa van Ee. The dissertation is clear, lucid and systematic in covering a very complex problem area. It contributes to increasing our knowledge and understanding of how severely traumatized refugee parents affect their non-traumatized children. New questions have arisen which can lead to further important research and help us find new ways and strategies in clinical treatment. Severely traumatized asylum seekers or refugee parents and their non-traumatized children are vulnerable groups and the numbers are increasing in our world today. This is why it is important to have further research following Elisa van Ee's important dissertation. 